Dahlia Shehata

\title{
Eine mannshohe Leier im altbabylonischen Ištar-Ritual aus Mari (FM 3, no. 2)
}

DOI 10.1515/aofo-2017-0008

\begin{abstract}
The Old Babylonian Ištar ritual from Mari (FM 3, no. 2) has been the focus of much discussion since its primary edition in 1938 by G. Dossin. This article offers a new analysis of the passage mentioning the balaĝ-deity Ninigizibara, which leads to identifying this bala $\hat{g}$ as a huge upright lyre as tall as a human played by two persons from both sides. Similar musical instruments are known from Anatolia and Egypt. Especially the Egyptian examples, which are attested only for the time of Echnaton, show striking parallels to the musical performance described in the Old Babylonian Ištar ritual. After discussing the possible background of cultural exchange, this article closes with a revaluation and new interpretation of the term balaĝ.
\end{abstract}

Keywords: Musical instrument, lyre, Ištar ritual, bala $\hat{g}$-deity, Ninigizibara, Echnaton, İnandık

\section{Einleitung}

Als eines der wenigen, gut erhaltenen Beispiele einer Ritualbeschreibung aus dem 2. Jahrtausend v.Chr. erfuhr das altbabylonische Ištar-Ritual aus Mari RA 35, 1 ff. (=FM 3, $\left.n^{\circ} 2\right)$ bereits in der Vergangenheit vielfach Beachtung. Die jüngste Bearbeitung des Textes findet sich bei Ziegler (2007: 55-63), im Zusammenhang mit der Emesal-Kultpraxis wurde das Ritual zuletzt von Gabbay (2014b: 174 und passim) besprochen, ${ }^{1}$ Heimpel (2015: 612-615, 623) interessierte vornehmlich die im Ritual auftretende balaĝ-Gottheit Ninigizibara.

Ausgehend von der Angabe [r]ēš warhi in Kolumne ii $20^{\prime}$ wurde das Ritual wohl regelmäßig am Anfang eines jeden Monats durchgeführt. ${ }^{2}$ Den zeitlichen und musikalischen Rahmen der Darbietung bildete, wie von Cavigneaux (1998: 46) festgestellt, der Vortrag des Balaĝ-Liedes Uruamma’irabi. ${ }^{3}$

\section{Zu den Zeilen i 10'-16'}

Im Verlauf des chorischen und instrumentalen Vortrags des Balaĝ-Liedes Uruamma'irabi treten verschiedene Kultstatisten in Aktion. Die Vorbereitungen im Tempel sowie die Aufstellung der teilnehmenden Personen und Kultobjekte werden vor allem in den ersten zwei Kolumnen der Ritualbeschreibung genannt. Danach wurden im Tempel zunächst das vergöttlichte Musikinstrument Ninigizibara und das Kultbild der Ištar einander gegenüber gestellt. In den Zeilen i 10'-16' werden die Musiker genannt, die bei Ninigizibara sitzen bzw. stehen. Der Text ist an den Zeilenanfängen zerstört. Durand/Guichard und Ziegler ergänzen hier jeweils unterschiedlich:

1 S.a. Löhnert (2009: 63-67)

2 So Volk (1989: 18, 1 Anm. 8 und id. 2006: 37, 91); s.a. Gabbay (2014b: 160 Anm. 45) zum monatlichen Vortrag im seleukidischen Uruk.

3 Cavigneaux (1998: 46): „...on peut conclure, que toute la cérémonie évoquée dans le ritual de Mari tournait autour de ce même balaĝ, interrompu par la recitation de l'ersemma...“.

Dahlia Shehata, Lehrstuhl für Altorientalistik, Universität Würzburg, Residenzplatz 2, Tor A, 97070 Würzburg, Deutschland, E-Mail: dahlia.shehata@uni-wuerzburg.de 


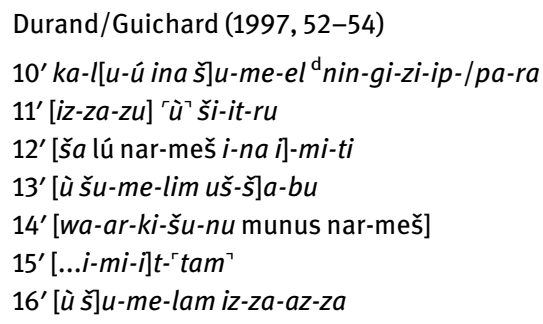

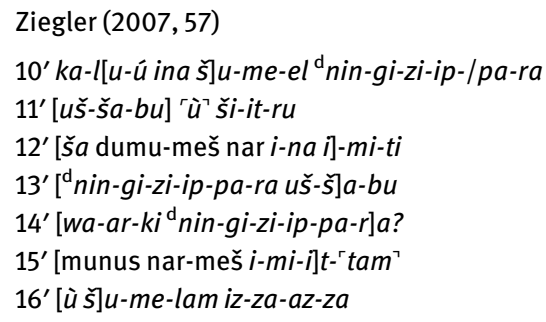

Zieglers Auffassung von imitti in $12^{\prime}$ als stat. constr. trägt m. E. entscheidend zum Verständnis dieser Zeilen bei. Danach wurde die Aufstellung der Klagepriester $\left(10^{\prime}\right)$ und der Orchester $\left(11^{\prime}\right)^{4}$ symmetrisch, nämlich links und rechts des Instruments Ninigizibara, vorgenommen. In den folgenden Lücken ergänzen Durand/ Guichard und Ziegler aufgrund der Form izzazzā eine Gruppe weiblicher Musiker, die sich bei Ziegler ebenfalls links und rechts der von ihr rekonstruierten balaĝ-Gottheit Ninigizibara aufstellen. Die Ergänzung weiblicher Musikerinnen ist allerdings zu bezweifeln, da solche im weiteren Ritualtext nirgends genannt werden und die Anwesenheit von Musikerinnen beim Ritual ohne Funktion eher unwahrscheinlich ist.

Den Schlüssel zum Verständnis dieser Zeilen liefert dennoch die Form izzazzā. Allerdings ist sie nicht als Plural f., sondern als Dual aufzufassen. Damit bezieht sich die Verbalform auf nur zwei Musiker, und zwar auf die Spieler des Instruments Ninigizibara, die sich links und rechts an seine Seiten aufstellen. Ich ergänze daher wie folgt:

\section{RA 35, $1 \mathrm{ff}$. Kolumne i}

\author{
Transliteration \\ $10^{\prime}$ ka-l[u-ú ina š $] u-m e-e{ }^{\mathrm{d}}$ nin-gi-zi-ip-/pa-ra

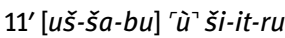 \\ $12^{\prime}[$ ša NN dumu-meš nar-meš $i-n a i]-m i-t i$ \\ 13' [ ${ }^{\mathrm{d}}$ nin-gi-zi-ip-pa-ra uš-š] $a-b u$ \\ $14^{\prime}$ [i-na i-di ${ }^{\mathrm{d}}$ nin-gi-zi-ip-pa-r]a \\ $15^{\prime}$ [2 ka-lu-ú i-mi-i]t-'tam? \\ 16' [ù š]u-me-lam iz-za-az-za
}

\author{
Übersetzung \\ Die Klagepriester sitzen links von \\ Ninigizibara und die Orchester, \\ [bestehend aus ... -Musikern], \\ sitzen rechts von Ninigizibara. \\ [An den Seiten der Ninigizibar]a \\ stehen links und re[chts \\ zwei Klagepriester].
}

\section{Anmerkungen zum Text}

12': In der Lücke ist eine nähere Spezifizierung der Orchester zu erwarten. Da das šitrum generell aus Musikern oder Musikerinnen besteht (in FM 9, 12 munus-meš, ARM 13, 22 und FM 9, 41 aštalêtum), sollte an dieser Stelle mehr als nur die Angabe von Musikern stehen, wie von Durand/Guichard und Ziegler ergänzt. Zu erwägen wäre daher entweder eine Angabe a) zur Anzahl der beteiligten Musiker (so in FM 9, 12: 10, 15 : 11 und 50: 9), b) zu den gespielten Instrumenten (nur indirekt in ARM 10, 137: 13) oder c) die Zugehörigkeit zu einem


könnte auch der Name eines Tempels zu ergänzen sein). Nach Kol. ii 25’ wird dieselbe Gruppe von Musikern aus dem Tempel zum Empfang der Läufer hinausgeführt; vgl. Ziegler $(2007: 58,61)$ zur Lesung der Zeile.

15': Für die Ergänzung des Klagepriesters kalû als Spieler des balaĝ sprechen mehrere Indizien. ${ }^{5}$ So ist für den babylonischen Raum des 2. Jts. bekannt, dass mit der Wartung des balaĝ-Instruments der Oberklagepriester (gala-mah) betraut war (Shehata 2009: 162). Prominent ist außerdem die Passage im Fluch über Akkade, wonach ein gala-mah sieben balaĝ aufstellt und erklingen lässt. ${ }^{6}$ Nach einem Balaĝ-Lied des 1. Jts. spielte

4 Gabbay (2014b: 83) übersetzt šitru entgegen bisheriger Auffassung als „Chor“.

5 Zum kalû als Spieler des balaĝ s.a. Gabbay (2014b: 85-87).

6 Vgl. Cooper (1983: 58-59): ${ }^{200}$ bala ĝ imin-e an-úr gub-ba-ginn 7 ki mu-nu-ši-ib-ús ${ }^{201}$ ùb me-zé li-li-ìs ${ }^{d_{i s ̌ k} \text { ur-gin }} 7$ šà-ba mu-na-an-tuk „Sieben balaĝ platzierte er, wie eine (hoch) stehende Himmelsfeste; ub, meze und lilissu, spielte er für ihn 
auch der einfache kalû das balaĝ (Cohen 1988: 420 a+37-a+41; kollationiert bei Gabbay 2014b: 81 Anm. 1). Häufiger belegt ist - u.a. auch im hier behandelten Ištar-Ritual iii 16-18 -, dass kalû das šèm (Akk. halhallatum) spielten, nach Gabbay (2014b: 143-151) eine Rassel oder ein Sistrum, das ebenfalls zur Begleitung der Emesal-Liturgien eingesetzt wurde. Auch im aB Ištar-Ritual Nr. 3 Kol. iii 10' ist gegen Durand/ Guichard (1997: 60) nicht nar-meš, sondern KU.UŠ zu ergänzen, entsprechend der gängigen Schreibung im selben Text (so iv 16' dumu-meš KU.Uš), da auch hier davon auszugehen ist, dass zwei Klagepriester die Eršema-Klage der folgenden Zeilen iii 12-13 vortragen. Auch beim balaĝ handelt es sich um ein ausschließlich im Zusammenhang mit den Emesal-Liturgien genanntes Musikinstrument, weshalb als seine Spieler nur Vertreter der kalû-Profession in Frage kommen. Letztlich sei außerdem darauf hingewiesen, dass auf göttlicher Ebene nach literarischen Texten ausschließlich Göttinnen das balaĝ spielen. ${ }^{7}$ Auch dies verweist auf kalû-Priester als Spieler des balaĝ, da diese im Rahmen der Emesal-Kultrituale die Rolle der klagenden Göttin einnehmen. ${ }^{8}$ Von einer Ergänzung mit $k$ /galamahhu wird hier abgesehen, da in Mari bislang nur einfache kalû-Priester nachweisbar sind, die im Wirkungskreis der Palastadministration dem nargallum unterstellt waren (Ziegler 2007: 64-65).

Eine andere Meinung vertrat jüngst Heimpel (2015: 591-592), demzufolge nicht die Klagepriester, sondern der Harfenist bala $\hat{g}$-di das balaĝ spielte, das dementsprechend als Harfe zu identifizieren sei. ${ }^{9}$ Da die Beleglage zum Beruf des bala $\hat{g}$ - di, der meist von Frauen vertreten wird, für das 2. Jt. eher dürftig ist (Shehata 2009: 94-98), vermute ich, dass seine Aufgaben, insbesondere im Bereich der rituellen Klage, auf den gala / kalû übertragen wurden.

\section{Diskussion}

In der bisherigen Forschung wurde Ninigizibara, das personifizierte und vergöttlichte Musikinstrument balaĝ aus dem Kreis der Inanna/Ištar, meist als Leier, genau genommen als Kastenleier identifiziert. ${ }^{10}$ Die hier gebotene neue Ergänzung und Deutung der Zeilen i 14'-16' scheint dies nicht nur zu bestätigen, sie erlaubt zudem eine Korrelation mit der ikonografisch belegten mannshohen Kastenleier, die von zwei Seiten von zwei Personen gleichzeitig gespielt wurde.

Kastenleiern sind in Babylonien sowohl mit als auch ohne Stierkopfapplikationen, im Original und ikonografisch bis in die Anfänge des 2. Jts. gut belegt. Allerdings werden diese meist von einer einzelnen Person sitzend oder auch vor der Brust getragen gespielt (Rashid 1984: 28-45, 61 Abb. 36, 64-67, 91 Abb. 7879). Das beidseitige Spiel einer Leier ist für den mesopotamischen Raum m.W. bislang nur auf einem einzigen Uruk-zeitlichen Siegel aus Uruk dargestellt (Abb. 1). Reichhaltiger ist demgegenüber die Beleglage aus der Peripherie. Berühmt ist die Darstellung auf der İnandık-Vase (Abb. 2). Vergleichbare Abbildungen sind auch aus dem Amarna-zeitlichen Ägypten (Abb. 3a/b) bekannt. Mannshohe Standleiern, allerdings einseitig gespielt, sind außerdem auf ägäischer Keramik belegt (Abb. 4), die in die zweite Hälfte des 2. Jts. datiert.

(= Enlil) in ihrer Mitte“; gegen die gängige Wiedergabe von an - úr als „Horizont“ fasse ich úr mit seiner Bedeutung „Wurzel“ oder auch „Fuß“ an dieser Stelle als eine Art Stütze auf, die durch die hier genannten sieben bala $\hat{g}$ gebildet wird und bildlich den Himmel trägt. In übertragener Bedeutung wird hier sicher auch auf die Funktion der balaĝ-Gottheiten im Kult angespielt.

7 Dumuzi-Inanna $F^{37}\left[\mathrm{x} \mathrm{x}^{\mathrm{d}} \mathrm{E}\right] \mathrm{N}^{\text {?. }}$.LÍL *bala ĝ ér da-mu-ra-ša ${ }_{4}, \ldots$. zusammen mit dem balaĝ werde ich dich beklagen“ (Sefati

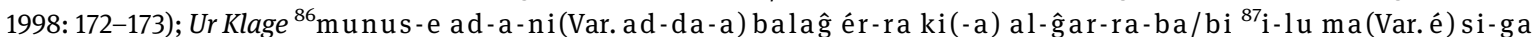
tur-tur-bi ní-te-na mi-ni-ib-bé „Nachdem die Frau (= Ningal) ihre Stimme?, das balaĝ der Klage, aufgestellt hatte; sprach sie zu sich selbst ein leises ,Wehe'-Lied über das schweigsame Haus“, vgl. Römer (2004: 27, 92, 112).

$8 \mathrm{Vgl}$. beispielsweise den Gestus des Brust(= Trommel)schlagens bei kalû wie auch bei der klagenden Muttergöttin, der auch ikonografisch belegt ist; Kilmer (1977: 133-135); Shehata (2014: 113-114); Gabbay (2014b: 142).

9 Zum gleichnamigen Instrument ba la $\hat{g}$ - di, Akkadisch timbuttu und weitere Varianten, s. jetzt Gabbay (2014b: 114-116).

10 Sallaberger (1993: 88 Anm. 374); Heimpel apud Cavigneaux (1998), „boeuf-lyre“ und Ziegler (2007: 60); zuletzt Gabbay (2014b: 105-106 passim). 


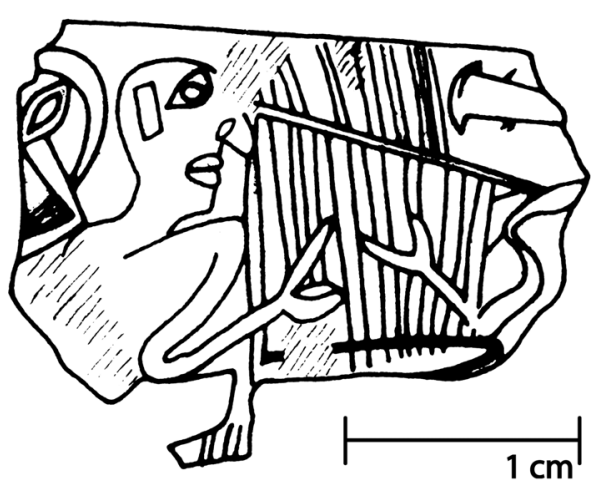

Abb. 1: Uruk-zeitliches Siegel mit beidseitig gespielter Leier; nach Boehmer (1992: 68).

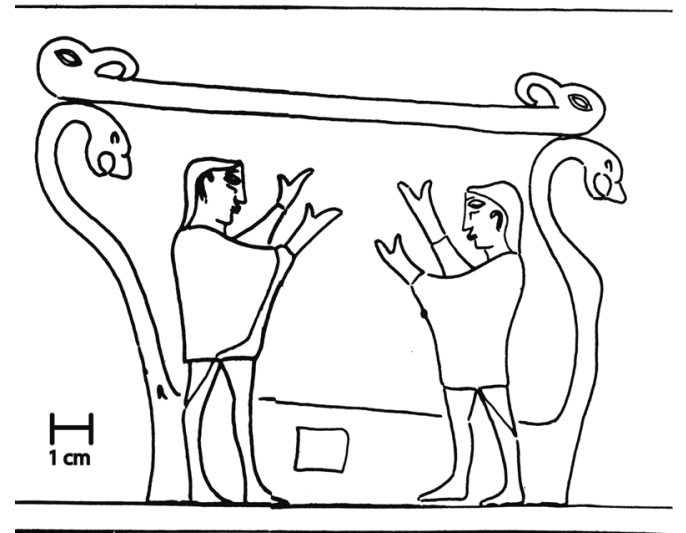

Abb. 2: Große, beidseitig gespielte Standleier der İnandık-Vase; nach Özgüç (1998: n174 Fig. 64).
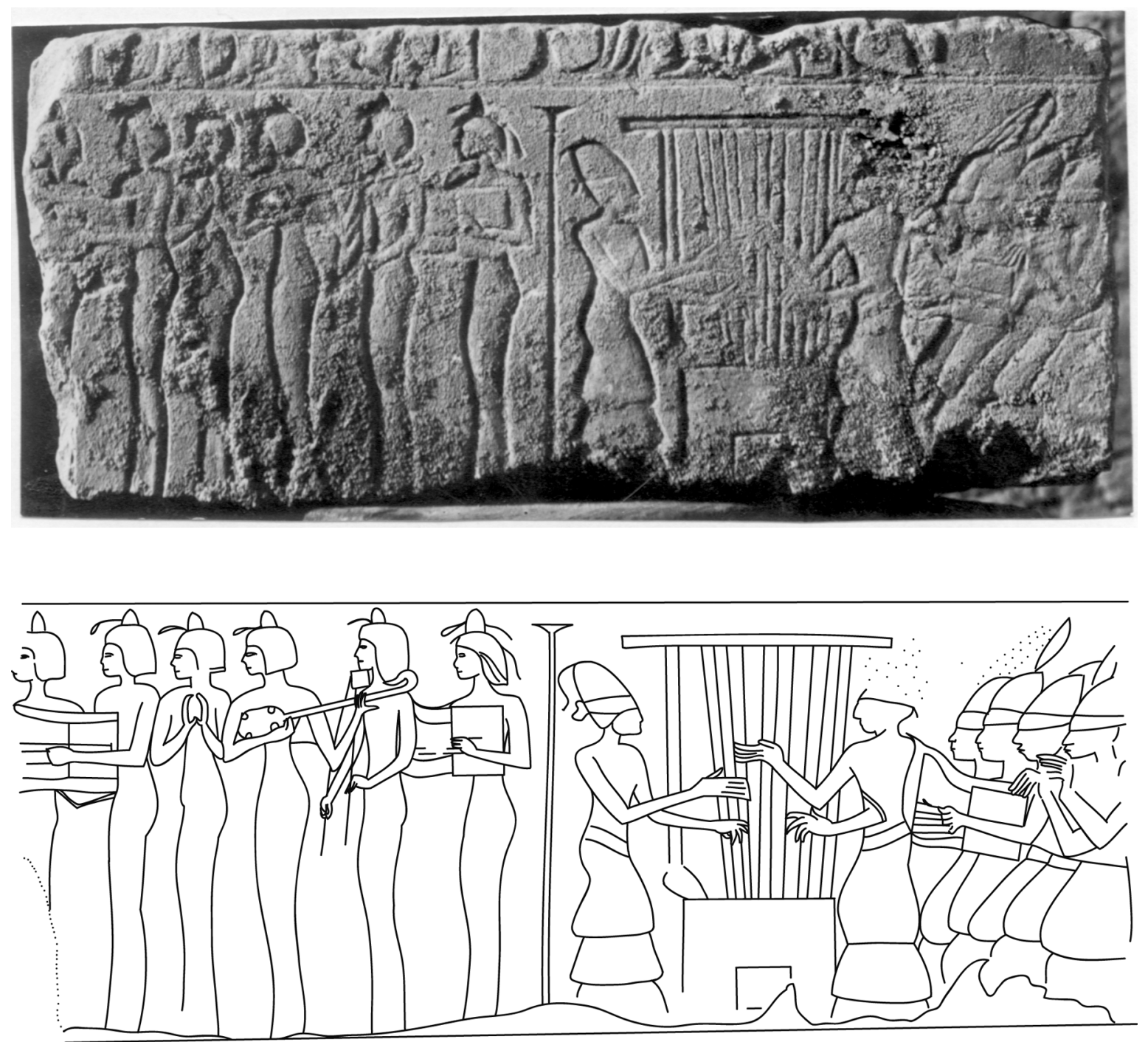

Abb. 3a1-Abb. 3a2: Beidseitig gespielte Standleier auf dem talatāt Nr. 21 des Aton-Tempels, Karnak, 18. Dynastie. Maße nicht ermittelbar; für die Bereitstellung des Fotos danke ich herzlich Lise Manniche. Umzeichnung D. Shehata. 


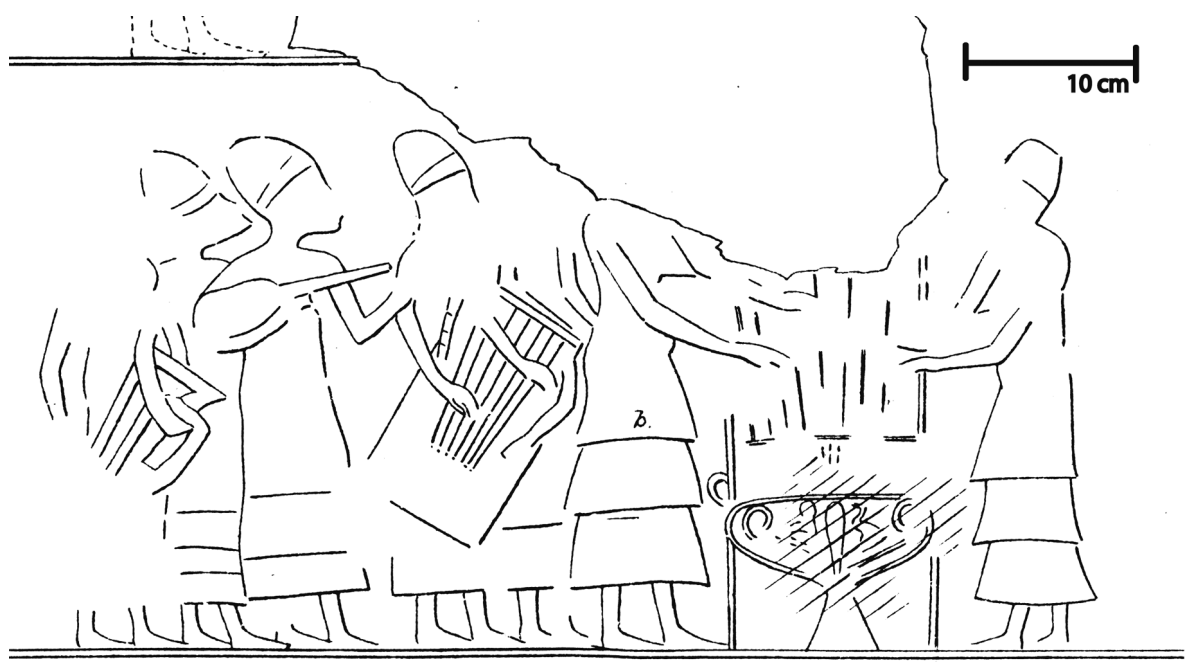

Abb. 3b: Beidseitig gespielte Standleier aus dem Grab des Huya, Tell el-Amarna, 18. Dynastie; nach Davies (1905: pl. VII).

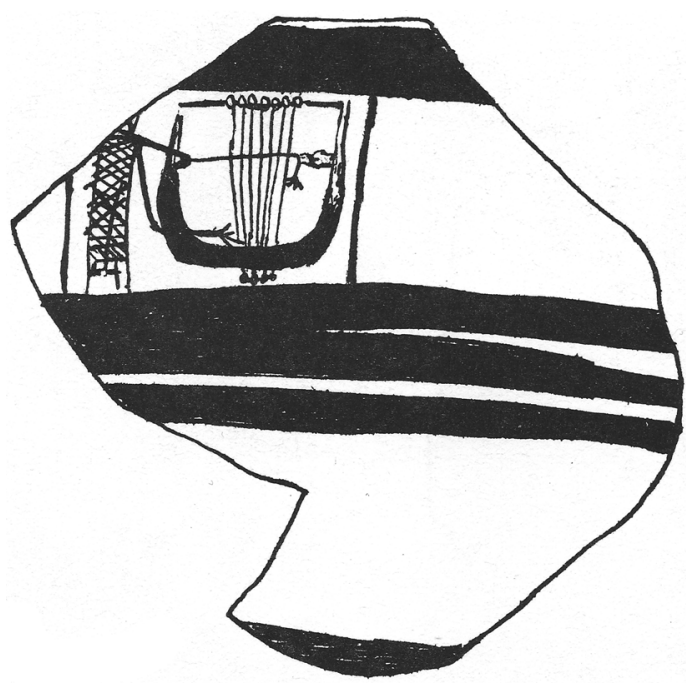

Abb. 4: Wand- und Randfragment eines Kraters mit Darstellung einer rundförmigen Standleier, die von einer einzigen Person gespielt wird, Nauplion (Mykene), Maße nicht ermittelbar; nach E. Vermeule/V. Karageorghis, Mycenaean Pictorial Vase Paintings, Cambridge, Harvard University Press (1982: X.14.1).

Das einzige weitere Instrument, das gleichfalls ausschließlich nach ikonografischer Evidenz von zwei Musikern beidseitig gespielt wurde, ist die große Rahmentrommel. Diese ist vor allem im 3. Jt. mannshoch, im altsyrischen Ebla (2. Jt.) sowie in Karkamiš (1. Jt.) fällt sie hingegen kleiner aus (Rashid 1984: 68-73; Matthiae 1987: 131, 150 Fig. 9). Die von zwei Personen gespielte Rahmentrommel wird inzwischen übereinstimmend mit dem in Texten belegten alû(m) (Sum. meist á-lá) identifiziert, das zuletzt ausführlich von Mirelman (2014) besprochen wurde. Besonders aussagekräftige Belege, was die enorme Größe des Instruments betrifft, finden sich in den altbabylonischen Mari-Briefen (Ziegler 2007: 74-76; Mirelman 2014: 155-156). So werden einmal für die Herstellung seines Klangkörpers 40 Minen Bronze benötigt, ein anderes Mal braucht es für den Transport eines alûm von Mari nach Aleppo 16-30 Männer (ARM 26/2, 286; ARM 26/1, 18 und 19).

Zwar überwiegen in der Forschung die Stimmen, denen zufolge das balaĝ Ninigizibara als Leier zu identifizieren sei. Was allerdings das Musikinstrument balaĝ betrifft, so scheint es nach etlichen Belegen auch 
ein Membranophon zu bezeichnen. ${ }^{11}$ Wie bereits von Kilmer (2003-2005: 369 §3.3) festgestellt, besteht dieselbe Ambivalenz in der möglichen Deutung auch für das alu $(m)$ (s.a. Mirelman 2014: 151). Im Folgenden werden daher auf dem Hintergrund unserer neuen Lesung erneut die Argumente zur Identifizierung von balaĝ und $a l \hat{u}(m)$ in Kürze skizziert. Anschließend wird der Frage nach Ursprung und Verbreitung des beidseitigen Spiels mannshoher Leiern nachgegangen.

\subsection{Ninigizibara: Leier, Harfe oder Trommel?}

Grundlage für die Identifizierung der Gottheit Ninigizibara als Leier lieferte ihre Einordnung als $\mathrm{gu}_{4}$-bala $\hat{\mathrm{g}}$ „Stier-balaĝ" in der Götterliste An=Anum IV 73-75 sowie in der Emesal-Liste I 87-88 (Litke 1998: 153-154; MSL 4, 9). Derselbe Ausdruck $\mathrm{gu}_{4}{ }^{\text {-bala }} \hat{\mathrm{g}}$ ist außerdem in der mA Liste KAV 64 ii 16 aus Assur belegt, ${ }^{12}$ ansonsten aber unbekannt. Dennoch wurde er mehrfach mit den berühmten, mit Stierkopf versehenen Prunkleiern aus den königlichen Gräbern von Ur in Verbindung gebracht, die in die erste Hälfte des 3. Jts. v. Chr. datieren und ikonografisch bis an das Ende desselben Jahrtausends belegt sind (Gabbay 2014b: 96). ${ }^{13}$ Dass eine solche Verbindung aufgrund der großen zeitlichen Distanz zwischen den Attestationen problematisch ist, bemerkte bereits Michalowski (2010: 220-222).

Für die Identifizierung des Instruments balaĝ als Leier spricht auch die Gleichung BALAĜ $=k \hat{i}-n a-r u_{12^{-}}$ um/lum/rúm aus Ebla (MEE 4, 264: 572) ${ }^{14}$ die zweifellos den westsemitischen Namen der Leier kinnāru wiedergibt. Doch auch diese Quelle datiert in das 3. Jt. v. Chr. In einer weitaus jüngeren Liste aus Emar wird das Wort kinnāru „Leier“ dem Ausdruck ${ }^{z a . d}$ MÜŠ geglichen (Emar 6/4, 76 Nr. 545: 392'). ${ }^{15}$ Als phonetischlogografische Schreibung scheint dieser den Instrumentennamen zannaru wiederzugeben. ${ }^{16}$ Dasselbe Instrument verbirgt sich wohl auch hinter dem hethitischen ${ }^{\mathrm{d}}$ INANNA/MÜŠ.(GAL/TUR) „(große/kleine) Inanna(-Leier)“, was ein der sumerisch-babylonischen Ninigizibara nicht unähnliches, kultisches Musikinstrument sein muss.

Auf ein Membranophon deuten wiederum Belege, die zum einen das Material des balaĝ betreffen. So wird in lexikalischen Listen ein „Ring“ (gúr // kippatu) des balaĝ genannt und auch das Hauptmaterial Bronze bzw. Kupfer lässt auf ein Schlaginstrument schließen (Ur 4 -ra VI 106; MSL 6, 60). ${ }^{17}$ Zum anderen wird der Klang des balaĝ zuweilen als besonders laut beschrieben. ${ }^{18}$

Nach Gabbay (2014a; 2014b: 93-102, auch zur Identifizierung des bala $\hat{g}$ ) ist die Mehrdeutigkeit des Terminus auf einen Wandel der Kultpraxis zurückzuführen. Seinen Beobachtungen zufolge sei das bala $\hat{g}$ spätestens seit dem 2. Jahrtausend mit der Kesselpauke lilissu zu identifizieren, während es in früheren Zeiten

11 Gelb (1975: 46, 57-58) auch zu früheren Deutungen; Black (1991: 9, 28-29); Veldhuis (1997/1998: 120); Cooper (2006: 41-42 Anm. 6) und Michalowski (2010: 206 Anm. 17 und S. 220).

12 Dort Akkadisch mundalku geglichen, zur theologischen Deutung des bala $\hat{g}$ als „Berater“ der ihm zugeordneten Gottheit s. jetzt ausführlich Gabbay (2014b: 103-109).

13 Die jüngste Darstellung einer Leier mit Stierkopf ist m.W. das Modell bei Rashid (1984: 66-67 Abb. 46), weiter besprochen bei Michalowski (2010: 231, Fig. 2a-c).

14 Hierzu zuletzt Franklin (2015: 65-67).

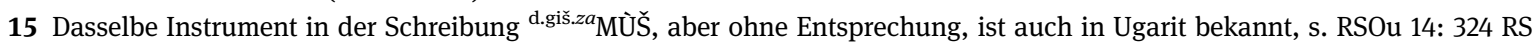
92.2004: 37 .

16 Das kinnāru(m) ist ausschließlich im syrischen Raum bezeugt, das zannaru dagegen ist wohl bereits im 3. Jt. v. Chr. in Mesopotamien bekannt; ausführlich zum zan(n)aru Krispijn (1990: 12); Richter (2004²: 293), Schuol (2004: 97-98) und zuletzt Franklin (2015: 76-79, 89-96).

$17 \mathrm{Zu}$ kupfernen bzw. bronzenen Instrumenten s. beispielsweise UET 3, 282 Vs. 15. ní $\hat{\mathrm{g}}$ - dab $\mathrm{d}_{5}$ bala $\mathrm{g}$ zabar (auch bei Heimpel 2015: 619: 35 genannt) und Kienast (1978/1, Nr. 32:14-15) gegen Kienast (ibid., 33) und Gabbay (2014b: 100) als bala $\hat{g}$ und nicht als lilis x zu lesen; s. weiter unten zur umfassenden Bedeutung des Begriffs bala $\hat{g}$. Die Erwähnung von kuš „Tierhaut“ im Zusammenhang mit bala $\hat{g}$, ob als Determinativ oder als sein Material, ist kein eindeutiges Indiz für seine Gattung, da Tierhäute sowohl für die Membran von Saiteninstrumenten wie von Membranophonen verwendet wurden; contra Gabbay (2014a: 136).

18 Vgl. beispielsweise die wie Stiere laut tönende Halle des/der? balaĝ-Instruments/e im Gudea Zyl. A xxviii ${ }^{17}$ a-ga balaĝ-a-bi gud gù nun-di. 
ein Saiteninstrument, höchstwahrscheinlich die Leier bezeichnete. ${ }^{19}$ Eine gänzlich andere Meinung vertrat jüngst Heimpel (2015), demzufolge das balaĝ, ausgehend von der ältesten bildlichen Gestalt des Zeichens, eine Harfe sei. ${ }^{20}$

Auch der Terminus alû( $m)$ scheint nicht ausschließlich die große Rahmentrommel bezeichnet zu haben. So lassen Belege aus Emesal-Texten vermuten, dass derselbe Terminus auch ein kleines, von einer Person zu tragendes Instrument meint (s.a. Gabbay 2014b: 152). In der Liste DIRI III erscheint es unter dem Eintrag ${ }^{50}[\mathrm{a}-1] \mathrm{u}={ }_{\text {ŝiš }}$ BALAĜ.. TUR $=$ alû (MSL 15, 138-139) zwischen anderen, eindeutig als Saiteninstrumente identifizierten Termini, etwa zannaru, kinnāru und sammû. Die Häufung von Saiteninstrumenten ist ein Indiz für das primäre Ordnungskriterium in diesem Listenabschnitt. Als „kleines balağ“ (ŝišs̆ noch in $\mathrm{Ur}_{4}$-ra VIIB 65 (MSL 6, 122) belegt. ${ }^{21}$ Ausgehend von seiner Einordnung und Beschreibung muss es sich bei diesem alû(m) um eine kleine Leier oder Harfe handeln. Für die Identifizierung der beidseitig gespielten Ninigizibara ist es allerdings nur sekundär von Bedeutung (s. weiter unten).

Die neue Deutung unserer Zeilen i 14'-16' im aB Ištar-Ritual bestätigt die vorherrschende Meinung, das balaĝ Ninigizibara als große Standleier zu identifizieren. Hierzu führt nicht zuletzt auch der Ausschluss aller übrigen möglichen Deutungen. So fehlen m.W. bislang Belege, wonach die Kesselpauke lilissu beidseitig gespielt wurde. ${ }^{22}$ Dasselbe gilt für die Identifizierung des balaĝ als Harfe, die schon aufgrund ihrer Form gar nicht von zwei Personen gleichzeitig gespielt werden kann. Da Ninigizibara nie als alû(m) angesprochen wird, kann auch seine Identifizierung als große, beidseitig gespielte Rahmentrommel ausgeschlossen werden.

\subsection{Kulttransfer}

Mit der Identifizierung der Ninigizibara als große Kastenleier und seiner Korrelation mit den oben genannten Darstellungen aus dem Amarna-zeitlichen Ägypten (Abb. 3a/b) sowie der İnandık-Vase (Abb. 2) liegt der Nachweis vor, dass übermannshohe Kastenleiern auch in Syrien und wohl auch in Babylonien gespielt wurden, auch wenn es hierfür bislang nur einen einzigen bildlichen Beleg aus der Uruk-Zeit gibt (Abb. 1 und s.o.).

Sowohl die übermannshohe Kastenleier von İnandık (Ende des 16. Jhs.) als auch die entsprechenden Instrumente auf den talatāt des Karnaktempels (14. Jh.) müssen im Rahmen von Kultimporten eingeführt worden sein, da sie im einheimischen Bildinventar ansonsten nicht vorkommen. ${ }^{23}$ Im Falle Ägyptens bestätigen dies zudem die ausländischen Musiker bzw. Priester, von denen zwei beidseitig das Instrument spielen, und eine andere geschlossene Gruppe - wohl ein Chor - daneben sitzt (Manniche 1991: 91 Fig. 54). Dass es sich um Fremdländer handelt, ist nach Manniche (1991: 90) vor allem an ihrer für Ägypten sonst untypischen Bekleidung festzumachen..$^{24}$ Sie tragen einen langen Rock in drei Falten und eine hohe, konisch zulaufende Kopfbedeckung. Dieselbe übermannshohe Kastenleier ist auf vier weiteren talatāt des Karnak-Tempels sowie in mehreren Gräbern von Tell el-Amarna abgebildet (Manniche 1974: 88-89). ${ }^{25}$

19 Gabbay (2014b: 94) „In my opinion, the available textual and iconographical evidence demonstrates that originally the bala $\hat{g}$ was a stringed instrument, probably a lyre, and that with time - probably towards the end of the third millennium - the term began to refer to a drum as well.“ (Hervorhebung durch die Autorin).

20 Auf der anderen Seite werden weitere mögliche Identifizierungen des bala $\hat{g}$ bei Heimpel (2015) vorgeschlagen, so ist es ibid. (S. 573-576) eine Leier in Ebla, eine Laute bei Gudea und ibid. (S. 579) eine Kesselpauke in Ur.

21 S.a. CAD A/1, 377b alû C; als „kleines balaĝ““ (ĝiš Diri III 51 (MSL 15, 138); vgl. CAD S $4 a$.

22 Trotz seiner These hält auch Gabbay (2014b: 99 Anm. 152) an der Identifizierung der Ninigizibara als Leier fest.

23 S. zu Musik- und Kulttransfer, ganz explizit zum vergöttlichten Instrument knr im Zusammenhang zur zypriotischen Figur des Kinyras, jetzt ausführlich Franklin (2015).

24 S. jetzt auch Franklin (2015: 108) als „three-tiered Levantine robes“ identifiziert.

25 Manniche unterscheidet zwischen Standleiern mit kasten- und solchen mit vasenförmigem Resonanzkasten; bei zwei der Abbildungen ist m. E. die Unterscheidung nicht eindeutig möglich; Davies (1905: pl. VII [Grab des Huya]) und id. (1908: VI pl. VI. [Grab des Parennefer]). 
Einen näheren Blick verdient an dieser Stelle die Szene auf dem Karnak-Relief von Abb. 3a. Besonders erstaunen hieran etliche Vergleichspunkte zur Aufstellung der Musiker im aB Ištar-Ritual. So sind auch auf dem Karnak-Relief zwei Gruppen von Musikern links und rechts der übermannshohen Kastenleier platziert. Die eine Gruppe wird von weiblichen Musikerinnen gebildet, die verschiedene Instrumente in Händen halten und damit durchaus als šitrū „Orchester“ zu identifizieren sind. Die andere sitzende Gruppe besteht aus den ausländisch gekleideten Priestern. Hierzu vergleichbar ist wieder das aB Ištar-Ritual 10'-11' kal[û ina š] umēl Ninigizippara [ǔ̌šabū] „Die kalû-Priester sitzen links von Ninigizibara“. Die Mitte bildet auf dem Relief die große Kastenleier, die von beiden Seiten von denselben Männern gespielt wird. Die zahlreichen Parallelen zwischen der Ritualbeschreibung aus Mari und dem Karnak-Relief, aber auch die auffällige Kleidung der auf Letzterem abgebildeten Priestermusiker samt hoher, konisch zulaufender Kopfbedeckung, verleiten zu dem Schluss, sie als kalû zu identifizieren, oder zumindest als Priester mit ähnlichem Status und Funktion. ${ }^{26}$

Auf der anderen Seite sind zwischen beiden Aufstellungen auch Unterschiede zu bemerken. So besteht die Musikergruppe rechts von der Leier (im Bild links) auf dem Karnak-Relief aus Frauen. In Mari werden als Spieler der Orchester rechts von Ninigizibara Gruppen männlicher Musiker rekonstruiert (i 12'). ${ }^{27}$ Unter der Gruppe der sitzenden Priestermusiker findet sich weiterhin ein einzelner, der eine kleine Leier in Händen hält.. ${ }^{28}$ Im Ištar-Ritual ist dagegen unter den kalû ein Spieler des halhallatum zu vermuten, da dieser im weiteren Verlauf des Rituals sich aus der Gruppe der sitzenden Priester erhebt, um, begleitet durch sein Instrument, ein Eršema solistisch vorzutragen (Ziegler 2007: 59: 16-18). ${ }^{29}$ Beachtenswert in diesem Zusammenhang ist wiederum eine Passage in Tafel 19 des Balaĝ Uruamma'irabi, wonach kalû mit dem alû bei der Göttin sitzen (Volk 1989: 18, 81, 91: 48-50). Angesichts der oben angeführten Belege ist hier sicher die alu $(m)$ Leier zu vermuten. Damit scheinen in der Beschreibung aus dem Balaĝ Uruamma'irabi aus dem 1. Jt. auch wieder Analogien zur Darstellung auf dem Karnak-Relief zu bestehen.

Ein letzter, aber wesentlicher Unterschied zwischen den Ritualbeschreibungen und dem ägyptischen Relief sind schließlich die gebundenen Augen der ausländischen Priester. Da m.W. hierzu keine Parallelen aus Mesopotamien bekannt sind, könnte der Grund hierfür gerade in der Fremdheit der ausländischen Priestermusiker zu suchen sein. Möglicherweise wurde es ihnen verwehrt, den Blick auf die ägyptische Gottheit zu richten..$^{30}$ Um diese Frage zu klären, wären Untersuchungen von ägyptologischer Seite notwendig.

Auf der İnandık-Vase ist die große, von zwei Personen gespielte Standleier im untersten Relieffries abgebildet. Rechts neben ihr schreitet ein Musiker mit Tragleier von ihr weg in Richtung zweier, einander gegenüber sitzender Figuren, die Özgüç (1988: 86-88) in ausführlicher Beschreibung als Götter deutet. Form und Gestalt der großen Standleier sowie der kleinen, tragbaren Leier sind miteinander identisch. Ihre Verzierungen mit nach hinten gerichteten Entenköpfen am Querjoch weisen sie dem ägyptisch-ägäischen Raum zu (Schuol 2004: 57-58). ${ }^{31}$ Abgesehen von der Gestalt der Leier sind keine Hinweise erkennbar, die auf einen fremden Kult hinweisen.

26 Zur Kopfbedeckung von kalû und anderen Priestern im 1. Jt. s. Gabbay (2014b: 74-75); in späthellenistischer Zeit kleideten sich kalû mit dem lubāru (UVB 1, 40: 10'-15'), das nach Zawadzki (2006: 91) ansonsten nur für Götter belegt ist. In der Nähe des Kultinstruments lilissu hatte der kalû allerdings ein TÚG.KUR.RA anzulegen (Textstellen auch bei Gabbay $2014 \mathrm{~b}: 74$ besprochen). Leider lässt sich bislang keines dieser Kleidungsstücke auf Abbildungen identifizieren.

27 Hierzu ist zu bemerken, dass die meisten in Mari-Briefen genannten Orchester (šitrū) aus Frauen bestehen, s. Ziegler (2007: 14). Gegen die Rekonstruktion eines weiblichen Orchesters spricht dennoch, dass keine Musikerinnen im weiteren Verlauf des Rituals genannt werden (s. o.).

28 So auch bei denselben Musikergruppen im Grab des Huya; Davies (1905: pl. V und VII), bei Letzterem sitzt unter ihnen außerdem ein Lautenspieler.

29 In einem zweiten Ritual aus Mari wird das Eršema von zwei kalû vorgetragen; Durand/Guichard (1997: 60): ${ }^{i i i} 10^{\prime} 2 \mathrm{du} \mathrm{m} \mathrm{[u]} \mathrm{-} \mathrm{m}$

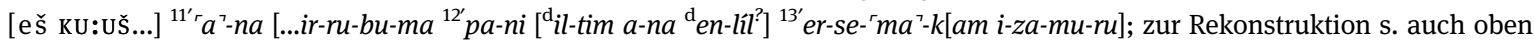
sub 2. Anmerkungen zum Text zu Zeile 15'.

30 Andererseits ist das Verbinden der Augen für viele Kulturen und Epochen im Ritus bei Grenzüberschreitungen bzw. einem symbolischen Übertritt in eine andere Welt bekannt; s. zu diesem Thema auch Manniche (1978, v.a. S. 20).

31 S.a. Rekonstruktionszeichnung Özgüç (1988: fig. 64 nach S. 174). 
Die kleine und große Leier werden von Schuol (2004: 102-106) nach De Martino (1987: 180-184 und

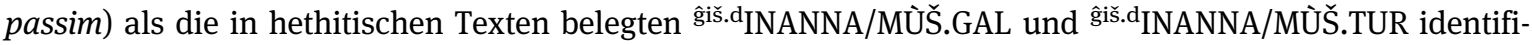
ziert. Wie auch immer die logografische Schreibung aufzuschlüsseln ist, so weist sie nach Weeden auf einen mesopotamischen Ursprung hin, möglicherweise sogar konkret auf die Stadt Uruk bzw. ihre Göttin Inanna (Weeden 2011: 252). ${ }^{32}$ Zwar ist die große Leier ikonografisch für den hethitischen Raum bislang nur einmal

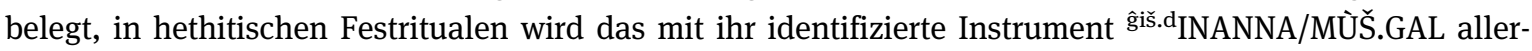
dings recht häufig eingesetzt, u. a. zur Begleitung von Totenzeremonien.

Der İnandık-Fund, der auch die berühmte Reliefvase beinhaltet, wird inzwischen in das letzte Viertel des 16. Jahrhunderts datiert. Für die Reliefvase kann nach Mielke (2006: 263-264) aufgrund ihres besonderen Wertes sogar ein etwas älteres Datum angesetzt werden, sodass sich die künstlerische Gestaltung ihres Bildprogramms selbst nach Neuer Chronologie und dem Ende Babylons um 1499 v. Chr. mit den Regierungsdaten der letzten Könige Babylons überschneiden würde. ${ }^{33}$ Die ägyptischen Reliefs aus dem Karnak-Tempel datieren bis zu zwei Jahrhunderte jünger in das 14. Jh. v. Chr. ${ }^{34}$

Auch wenn es sich bei der Leier der İnandık-Vase sicher um ein importiertes Musikinstrument handelt, so spricht die Beleglage eher für eine längere Existenz von Kulten um die große Standleier im anatolischen Raum. Möglicherweise wurden diese sogar noch vor den Eroberungszügen des Hattušili I. ins Land getragen. Setzt man drei Generationen für die Akkulturation an, so wäre die Übernahme spätestens für den Anfang des 18. Jhs. v. Chr. denkbar, also unter dem Einfluss des Kulturkontakts zur Zeit der altassyrischen Handelskolonien. Die ägyptisch anmutenden Leierverzierungen an den Jocharmen sind sicher über den palästinensisch-syrischen Raum nach Anatolien getragen worden..$^{35}$ Das mit Inanna assoziierte Instrument scheint dabei eine weite kultische Verbreitung gefunden $z u$ haben, was auch an jüngeren Belegen aus Emar und Ugarit nachvollziehbar ist (s. o.).

Gänzlich anders verhält es sich mit den Darstellungen auf den Amarna-zeitlichen Tempel- und Grabreliefs. Sowohl die mannshohe Kastenleier, als auch die dazugehörigen Musiker sind ein Phänomen, das auf die Regierungszeit Echnatons beschränkt bleibt. Es muss sich daher um einen Kult handeln, den dieser Pharao aus Syrien oder Babylonien für seinen neuen Gott Aton eingeführt hat, ganz offensichtlich zusammen mit den zugehörigen Priestern. Hier fallen außerdem etliche Vergleichspunkte zu kalû-Riten im syrischen sowie im jüngeren babylonisch-assyrischen Raum auf. Zwar ist insgesamt der Import von Fachpersonal, insbesondere von Musikern, nichts Ungewöhnliches. ${ }^{36}$ Eine Besonderheit ist allerdings der Einsatz von fremdem Personal im Kult sowie die Beschränkung dieses Phänomens auf die Regierungszeit des Echnaton. Von einem Zusammenhang mit dem neu eingerichteten Aton-Kult ist daher sicher auszugehen.

Abgesehen davon, dass etliche Fragen im Raum stehen, etwa aus welchem Grund und unter welchen Umständen diese Riten nach Ägypten kamen, welches Ritual oder Kultfest tatsächlich ausgeführt wird und um welches vergöttlichte balaĝ es sich dabei handeln könnte, ${ }^{37}$ führen die Beobachtungen letztlich zu dem Schluss, dass es entsprechende Kulte und Rituale, die die mannshohe Kastenleier integrieren, auch in Mesopotamien gegeben haben muss. Der m.W. jüngste Beleg für das vergöttlichte balaĝ Ninigizibara im Rahmen einer Kulthandlung liegt mit der Ausgabenliste MHET 1/1, 63 vor, die in die Zeit des gala-mah Inanna-mansum, Vater des berühmten Ur-Utu datiert, also vornehmlich in die Regierungszeit des Ammiditana (Mittlere Chronologie: 1683-1647; Neue Chronologie: 1586-1549 v. Chr.). Alle jüngeren Belege zur Ninigizi-

32 Für den Hinweis danke ich Michele Cammarosano.

33 Vgl. Übersicht bei Pruzsinszky (2009: 23-29 und passim).

34 Zur ägyptischen Chronologie im 2. Jt. v. Chr. s. Zusammenfassung bei Breyer (2010: 54-55 und passim).

35 Breyer (2010: 67-79 und 432-433) konkret zu Transferwegen am Beispiel des Sphingenmotivs.

36 S. allgemein zu fremder Musik in Ägypten van Lieven (2008) mit Verweisen auf ältere Literatur; auch die Korrespondenzen aus Tell el-Amarna und Hattusa belegen letztlich den Austausch von unterschiedlichem Fachpersonal, etwa Ärzten oder Handwerkern, s. z. B. THet. 16, Nr. 204 Rs. i 34bff. oder ibid. 57bff. (ein Bildhauer).

37 Trotz des universellen Anspruchs des Aton-Kultes wäre es sehr ungewöhnlich, wenn ihm ein besänftigendes balaĝ, nämlich die abgebildete Kastenleier, zur Seite gestellt worden wäre. Vgl. hier auch die zwar fragwürdigen aber doch interessanten Analysen Forsyth' (1987: 55-57), der Parallelen zwischen den hymnischen Dichtungen an Ninurta und Aton beobachtet. Möglich ist auch, dass für den ägyptischen Aton-Kult ein passendes Instrument aus Mesopotamien abtransportiert wurde, schließlich wurde auch die ninivitische Šauška in dieser Weise ,ausgeliehen` (EA 23 bei Moran 1992: 61-63). 
bara entstammen Götterlisten sowie liturgischen und hymnischen Dichtungen (Heimpel 1998-2001: 382-384; Gabbay 2014b: 105-106). Es lässt sich also nicht sicher nachweisen, inwiefern die Leier Ninigizibara oder gar andere mannshohe Leiern noch eine aktive Rolle im Rahmen von Emesal-Kulten in nach-altbabylonischer Zeit gespielt haben.

\section{Zusammenfassung und Ausblick}

Wichtige Erkenntnisse aus der neuen Auslegung der Zeilen i 10'-16' im aB Ištar-Ritual sind die Folgenden:

1. Das vergöttlichte Musikinstrument Ninigizibara war kein passives Kultbild, sondern wurde zur Begleitung des chorischen Gesangs der balaĝ-Liturgie Uruamma’irabi eingesetzt. Damit nahm die mannshohe Leier aktiv am Ritual teil. ${ }^{38}$

2. Vergöttlichte Balaĝ-Instrumente können auch im 2. Jt. v.Chr. Leiern sein und nicht ausschließlich Trommeln oder Kesselpauken. ${ }^{39}$ Geht man davon aus, dass die im Karnak-Tempel dargestellten Szenen auf Kultimporte aktueller babylonischer Kulte zurückgehen, so wurden mannshohe Leiern noch in der 2. Hälfte des 2. Jahrtausends in Syrien und wohl auch in Babylonien im Kult eingesetzt.

3. Der Einfluss ursprünglich babylonischer Kultpraktiken reichte bis nach Anatolien und Ägypten. Identifiziert man die in Karnak dargestellten fremdländischen Priester als babylonische kalû, so wurden die Kulte sogar zusammen mit dem Tempelpersonal und ihrem wichtigsten Instrument importiert.

Weitaus zahlreicher sind die Fragen, die hieran anknüpfen und weiterführenden Studien vorbehalten sein müssen. So verwundert zunächst das gänzliche Fehlen schriftlicher wie bildlicher Belege zu mannshohen Kastenleiern aus dem mesopotamischen Raum für das 2. Jt. v. Chr. Ist dieses Schweigen mit einer lückenhaften Forschungs- und Quellenlage zu begründen? Oder wurde dieser Bereich der Tempelmusik aufgrund seiner Alltäglichkeit in Texten und Bildern einfach vernachlässigt? Auch wenn die importierten Kultpraktiken in Babylonien ihren Ursprung haben, so stellt sich dennoch die Frage, über welche direkten oder indirekten Wege sie nach Anatolien und nach Ägypten kamen. Problematisch bleibt außerdem noch die Identifizierung der auf den Karnak-talatāt und in den Amarna-Gräbern abgebildeten Priestermusiker. Hier wären dringend weitere vergleichende Untersuchungen notwendig, um mögliche Verbindungen zwischen den Kulten Babyloniens und des Amarna-zeitlichen Ägypten aufzudecken.

Schließlich fragt man sich, ob die für das 2. Jt. beobachtete Aufführungspraxis von Klageliturgien auch noch im 1. Jt. Geltung hatte. In diesem Zusammenhang ist auf eine Grabzeichnung aus dem ptolemäerzeitlichen Ägypten hinzuweisen (Abb. 5), die eine mannshohe, von einem einzigen Priester gespielte Kastenleier zeigt (Manniche 1974: 89). Beachtenswert ist der Stierkopf, der am rechten, dem Spieler zugewandten Ende des Querjochs zu sehen ist. Eine Verbindung zu den Stierleiern von Ur wurde bereits von Hickmann (1961: 34) und später von Schuol (2004: 104) aufgezeigt. Möglicherweise ist diese außerdem zu den mesopotamischen $\left(\mathrm{gu}_{4^{-}}\right)$bala $\hat{\mathrm{g}} \mathrm{zu}$ ziehen, die für das 1 . Jt. vor allem aus Götterlisten bekannt sind, in Ritualtexten derselben Zeit allerdings nicht genannt werden. Stets prominent in Tempelritualen des späten 1. Jahrtausends ist hingegen das lilissu (Gabbay 2014b: 118-139). ${ }^{40}$

38 So schon von Heimpel (1998-2001: 383a) vermutet, contra Gabbay (2014b: 87 und 98-99).

39 Vgl. Aussage bei Gabbay (2014b: 152 gegen ibid., 102).

40 Auch in spätbabylonischen Tempelritualen ist bislang einzig das lilissu nachweisbar; diesen Hinweis verdanke ich R. da Riva (September 2016). 


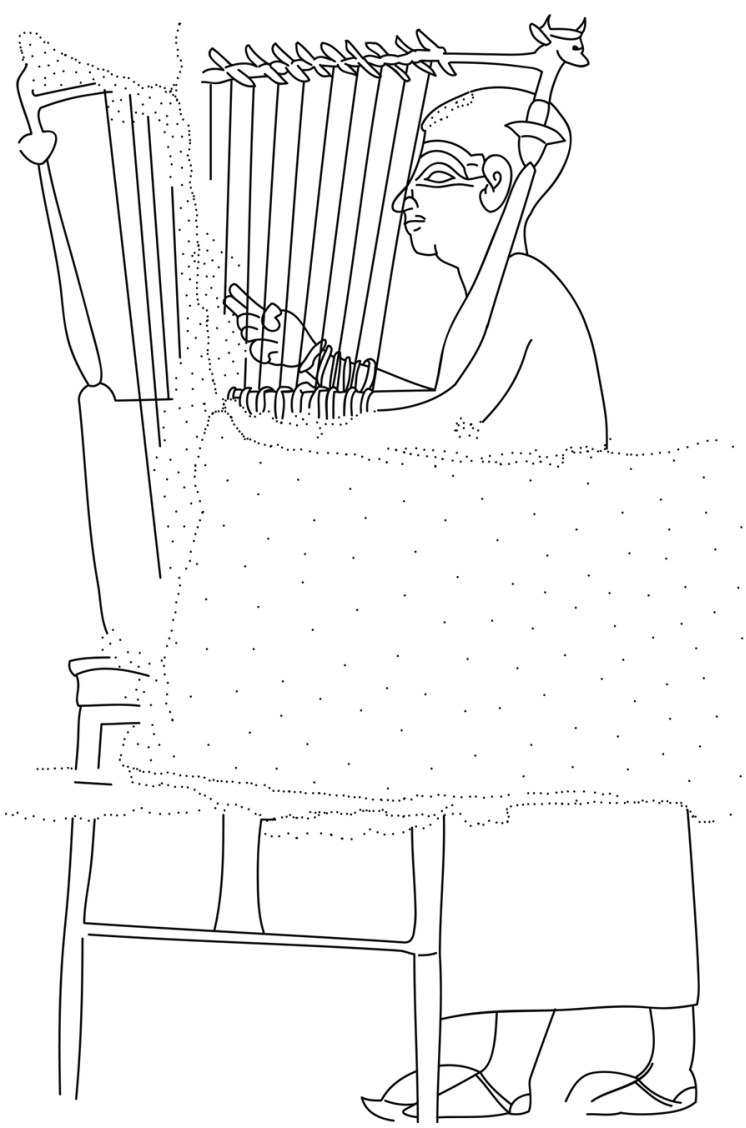

Abb. 5: Spieler einer Kastenleier mit Stierkopf am rechten Ende des Querjochs, Philae-Tempel (Ägypten), Ptolemäerzeit; nach Hickmann (1961: 35 Abb. 12).

Die Identifizierung antiker Musikinstrumente gestaltet sich mitunter sehr schwierig. Hier ist nicht nur die Vieldeutigkeit der Belege problematisch, sondern auch die unwirkliche Annahme, die moderne, vornehmlich von organologischen Kriterien bestimmte Einteilung der Instrumentengattungen ließe sich auf antike Systeme übertragen..$^{41}$

Was das balaĝ betrifft, so sind weder die Belege, die es als Membranophon ausweisen, noch diejenigen, denen zufolge es ein Saiteninstrument ist, von der Hand zu weisen. Da entsprechende Belege teilweise zeitlich parallel auftreten, stößt auch die These Gabbays zum Bedeutungswandel des Terminus im Laufe des 2. Jts. auf ihre Grenzen (s.o.). Aus diesem Grund möchte ich erneut an meiner bereits 2007 erstmals formulierten Hypothese festhalten, wonach das Wort balaĝ kein bestimmtes Instrument und auch keine Instrumentengattung bezeichnet. ${ }^{42}$ Es repräsentiert vielmehr ein religiöses Konzept, das auf der Vorstellung beruht, von Menschen erzeugte Musik könne Grenzen zu anderen Welten überschreiten und als Mittel der Kommunikation mit Göttern im Himmel oder auch Toten in der Unterwelt eingesetzt werden..$^{43}$ In dieser Funktion steht bala $\hat{g}$ für die höchst-mögliche und edelste Form der Musikpraxis. Der Terminus selbst verweist damit primär auf kultisch-funktionale und weniger auf organologische Merkmale eines Musikinstruments. Welcher Gattung die jeweiligen Musikinstrumente angehören, die diese Form der Kommunikation bewerkstelligen und in entsprechender Weise im Kult zum Einsatz kommen, ob es Membranophone oder Chordophone sind, ist dabei sekundär.

41 S.a. Franklin (2015: 532) ,the morphological difference between 'lyre' and 'harp', as defined by von Hornbostel and Sachs, may have been less significant than performance functions“.

42 Erstmals öffentlich auf dem SBL International Meeting in Wien 2007; s. außerdem Shehata (2014: 116-121, 123).

43 In eine ähnliche Richtung deutet Gabbay (2014b: 98). 
In seiner ganzen Komplexität und mit allen seinen theologischen Implikationen wird dieses Konzept des balaĝ in der Praxis erst im Laufe des 2. Jahrtausends konkretisiert und in gewisser Weise standardisiert. ${ }^{44}$ Hier wird es schließlich zur Grundlage der kalû-Theologie, wonach mithilfe der balaĝ-Klänge und der gleichnamigen Gesänge die Herzen unruhiger und erzürnter Götter besänftigt werden. ${ }^{45}$ Gleichzeitig wird hierdurch dieser musikpraktische Bereich auf die Expertise einer bestimmten Priestergruppe beschränkt. Inwiefern hiermit die seit dem 2. Jahrtausend stetig zunehmende Bedeutung des kalû-Priestertums sowie der im kultischen Bereich wachsende Einfluss seiner Mitglieder in Zusammenhang steht, wäre weiterführend in entsprechenden, diachron angelegten Studien zu untersuchen.

In seiner ursprünglichen Bedeutung könnte der Terminus balaĝ lediglich allgemein geordneten „Klang“ oder das „Musizieren“ bezeichnet haben. In diesem Sinne ist wohl auch die älteste Uruk-zeitliche Form des Piktogramms ZATU $47 \mathrm{zu}$ verstehen (Green/Nissen 1987: 179). Das Abbild der Harfe stellt hier nicht das konkrete Objekt Harfe dar. Vielmehr wird über dieses, in dieser Zeit am weitesten verbreitete Instrument wohl ganz allgemein musikalischer Klang abgebildet. ${ }^{46}$ Dass das spätere, frühdynastische Zeichen BALAĜ, auf das alle jüngeren Zeichenformen zurückgehen, schwer zu identifizieren ist, ${ }^{47}$ könnte damit zu begründen sein, dass es kein bestimmtes Musikinstrument darstellt, sondern möglicherweise eine bildliche Abstraktion des Musizierens oder gar des „Klangs“. ${ }^{48}$ Das alte Zeichen der Harfe wurde damit durch ein neues, der aktuellen Musikauffassung angepasstes Ikonograf ersetzt.

Personifiziert und vergöttlicht erscheint das oben beschriebene Konzept des balaĝ und seine Funktion im Kult als die Gottheit ${ }^{\mathrm{d}}$ balaĝ, die im ersten Jahrtausend in Götterlisten als der etymologisch unklare ${ }^{\mathrm{d}}$ Lumha erscheint, als „Ea der kalû“ erläutert wird und damit wieder den Bezug zur kalû-Theologie herstellt (Litke 1998: 104: 307 und 239: 131; Gabbay 2014b: 90). Gestalt und Gattung des Instruments, das für diesen bestimmten Bereich der kultischen Musikpraxis eingesetzt wird, unterliegen zum einen dem Wandel der Epochen und ihrer jeweiligen Musikästhetik, zum anderen Traditionen, die an regionale Spezifika oder auch an einzelne Gottheiten des Pantheons gebundenen sind. Ersteres geht konform mit der Beobachtung Gabbays, wonach im Verlauf des 2. und zunehmend im 1. Jahrtausend Kesselpauken diese Funktion im Kult übernahmen. Dass diese lilissu-Pauken als Repräsentanten der Funktion balaĝ verstanden wurden, zeigen altbabylonische Belege, in denen balaĝ gleich einem Determinativ dem Wort lilissu vorangestellt ist. ${ }^{49}$ Trotz dieser Entwicklung müssen dennoch, wie die hier behandelte Stelle zeigt, nicht alle balaĝ ab dem 2. Jahrtausend als Kesselpauken identifiziert werden. Schließlich zeugen die vielfältigen Namen der jüngeren $\mathrm{gu}_{4}$-bala $\hat{\mathrm{g}}$-Gottheiten von einer vielfältigen Gestalt und Klangvorstellung, die vor allem Charakteristika der ihnen zugeordneten Gottheit widerspiegeln ${ }^{50}$. Gemeinsames Merkmal ist das Element $\mathrm{gu}_{4}$ „Stier/Rind“, sei dieses im Namen zu finden oder als Referenz auf die Gestalt oder das Material, nämlich die Lederhaut, zu verstehen. Letztlich ist zu bezweifeln, dass tatsächlich alle in Götterlisten genannten $\mathrm{gu}_{4}$-bala $\hat{g}$ als konkrete Musikinstrumente existierten und im Kult gespielt wurden. ${ }^{51}$ Beschreibungen in Literatur und Ritualen zeigen deutlich, dass nur

44 Es spiegelt sich zum einen wider in lexikalischen „Übersetzungen“ des Terminus $\mathrm{g} \mathrm{u}_{4}$-bala $\hat{\mathrm{g}}$ als mundalku „Berater“ oder ad-gi - $_{4} \mathrm{gi}_{4}$, wörtlich etwa „Stimme zurückgeben“; vgl. Gabbay (2014a: 142).

45 Ausführlich Gabbay (2014b: 78-79, 103-109 und passim), allerdings mit anderer Deutung.

46 Dass das Bild der Harfe einen umfassenderen Begriff als das reine Objekt darstellen muss, bestätigt letztlich die Tatsache, dass in archaischen Texten nach bisheriger Kenntnis außer der Harfe keine weiteren Musikinstrumente vertreten sind.

47 Vgl. Krispijn (2002: 468, 477); Kilmer (2000: 115-119) und zuletzt Gabbay (2014b: 97-98); gängiges Beispiel für ein abstrahiertes Zeichen ist UDU; vergleichbar komplex ist auch das Zeichen EN.

48 Kulturübergreifende, vergleichende Studien zur bildlichen bzw. ikonografischen Wiedergabe von Klang oder Musik, aber auch anderer abstrakter Prinzipien, wären hier sicher weiterführend.

49 Im Jahresnamen Sumu-la-el d (BM 92615, CT 4, 50a) „Jahr: Sumu-la-el erbaute balaĝ (der Gestalt) lilis, zwei von ihnen, für Šamaš“ mu su-mu-la-èl balaĝ li-li-ìs 2-a-bi <d >utu-ra mu-na-an-dím; außerdem in der Inschrift Warad-Sîn 35 (RIME 4, S. 460) iii

${ }^{8}$ 'balaĝ [1]i-li-ìs ... ${ }^{\prime}$ mu-[n]a-dím, wo das balaĝ seinem Vater Kudurmabuk geweiht ist, s.a. die Hammurapi-Inschrift RIME 4 3.6.11:

${ }^{31}$ balaĝ li-l[i-ì]s zabar; hierzu bereits Gabbay (2014b: 99) mit weiterem Beleg, allerdings mit anderer Deutung.

50 Während beispielsweise für männliche Kriegergestalten wie der Wettergott Adad lautstarke balaĝ-Instrumente zum Einsatz kamen, wird das balaĝ der Ištar Ninigizibara durch die eher sanft tönende, aber wohl dennoch im Klang starke große Leier repräsentiert; vgl. Gabbay (2014b: 113-114).

51 Kaum als Musikinstrumente vorstellbar sind beispielsweise die $\mathrm{gu}_{4}$-bala $\hat{g}{ }^{\mathrm{d}}$ ní $\hat{\mathrm{g}}$-na „Räucherbecken“ und dgi-izi-lá „Fackel“ des Gibil (Litke 1998: 108: 343-344) oder die „Laus“ ( ${ }^{\mathrm{d}}$ up-lum) des Manungal (ibid. 186-187: 199-200); zu erwägen wäre 
wenige tatsächlich zum Einsatz kamen, am häufigsten belegt ist im 2. Jahrtausend die Ninigizibara-Leier, was sicherlich mit der Prominenz der ihr zugeordneten Gottheit Inanna/Ištar zu begründen ist.

\section{Bibliografie}

Black, J. A. (1991): Eme-sal Cult Songs and Prayers, AulaOr. 9, 23-36.

Boehmer, R. M. (1992): Von zwei Musikanten gespielte Leiern. In: H. Otten et al. (ed.), Hittite and other Anatolian and Near Eastern Studies in Honour of Sedat Alp (Türk Tarih Kurumu Basımevi), Ankara, 67-68.

Breyer, F. (2010): Ägypten und Anatolien: Politische, kulturelle und sprachliche Kontakte zwischen dem Niltal und Kleinasien im 2. Jahrtausend v. Chr. (Denkschriften der Gesamtakademie 63/Contributions to the Chronology of the Eastern Mediterranean 25), Wien.

Cavigneaux, A. (1998): Sur le balag Uruamma'irabi et le Rituel de Mari, NABU 1998/43.

Cohen, M. E. (1988): The Canonical Lamentations of Ancient Mesopotamia, Vol. 1/I2, Potomac, Maryland.

Cooper, J. S. (1983): The Curse of Agade, Baltimore u. a.

Cooper, J. S. (2006): Genre, Gender, and the Sumerian Lamentation, JCS 58, 39-47.

Davies, N. de G. (1905): The Rock Tombs of El Amarna. Part 3: The Tombs of Huya and Ahmes, London.

Davies, N. de G. (1908): The Rock Tombs of El Amarna. Part 6: Tombs of Parennefer, Tutu, and Ay, London.

De Martino, S. (1987): Il lessico musicale ittita II: GIŠ dINANNA = cetra, OrAnt. 26, 171-185.

Dossin, G. (1938): Un rituel de culte d'Ištar provenant de Mari, RA 35, 1-13.

Durand, J.-M./M. Guichard (1997): Les rituel de Mari. In: J.-M. Durand et al. (ed.), Recueil d’études à la mémoire de Marie-Thérèse Barrelet (FM 3 = Mémoires de N.A.B.U. 4), Paris.

Forsyth, N. (1987): The Old Enemy Satan and the Combat Myth, Princeton.

Franklin, J. C. (2015): The Divine Lyre (Hellenistic Studies 70), Washington DC.

Gabbay, U. (2014a): The Balaĝ Instrument and Its Role in the Cult of Ancient Mesopotamia. In: J. Goodnick Westenholz et al. (ed.), Music in Antiquity: The Near East and the Mediterranean (Yuval 8), Jerusalem, 129-147.

Gabbay, U. (2014b): Pacifying the Hearts of the Gods: Sumerian Emesal Prayers of the First Millennium BC (HES 1), Wiesbaden. Gelb, I. J. (1975): Homo ludens in Early Mesopotamia (StOr. 46), Helsinki, 43-76.

Green, M. W./H. J. Nissen (1987): Zeichenliste der archaischen Texte aus Uruk (ATU 2 = ADFU 11), Berlin.

Heimpel, W. (1998-2001): Ninigizibara I und II, RIA 9, 382-384.

Heimpel, W. (2015): Balang-Gods, apud J. C. Franklin (2015), 571-632.

Hickmann, H. (1961): Ägypten. Musikgeschichte in Bildern. Band 2 (Musik des Altertums, Lieferung 1), Leipzig.

Kienast, B. (1978): Die altbabylonischen Briefe und Urkunden aus Kisurra (FAOS 2/1-2), Wiesbaden.

Kilmer, A. D. (1977): Notes on Akkadian uppu. In: M. Dejong Ellis (ed.), Essays on the Ancient Near East in Memory of Jacob Joel Finkelstein (MCAAS 19), Hamden, Conneticut, 129-138.

Kilmer, A. D. (2000): Continuity and Change in the Ancient Mesopotamian Terminology for Music und Musical Instruments. In: E. Hickmann et al. (ed.), Musikarchäologie früher Metallzeiten (Studien zur Musikarchäologie 2 = Orient-Archäologie 7), Rahden (Westfalen), 113-119.

Kilmer, A. D. (2003-2005): Pauke und Trommel. A. In Mesopotamian, RIA 10, 367-371.

Krispijn, Th. J. H. (2002): Musik in Keilschrift: Beiträge zur altorientalischen Musikforschung 2. In: E. Hickmann et al. (ed.), Archäologie früher Klangerzeugung und Tonordnung (Studien zur Musikarchäologie 3 = Orient-Archäologie 10), Rahden (Westfalen), 465-479.

Litke, R. L. (1998): A Reconstruction of the Assyro-Babylonian God-Lists, AN: ${ }^{\mathrm{d} A-n u-u m ~ a n d ~ A N: ~ A n u ~ s ̌ a ́ ~ a m e ̄ l i, ~ N e w ~ H a v e n . ~}$ Löhnert, A. (2009): „Wie die Sonne tritt heraus!“: Eine Klage zum Auszug Enlils mit einer Untersuchung zu Komposition und Tradition sumerischer Klagelieder in altbabylonischer Zeit (AOAT 365), Münster.

Manniche, L. (1974): Ancient Egyptian Musical Instruments (Münchner Ägyptologische Studien 34), München.

Manniche, L. (1978): Symbolic Blindness, Chronique d’Égypte 53,13-21.

Manniche, L. (1991): Music and Musicians in Ancient Egypt, London.

Matthiae, P. (1987): Les dernières découvertes d’Ebla en 1983-1986, CRAIBL 131, 135-161.

Michalowski, P. (2010): Learning Music: Schooling, Apprenticeship, and Gender in Early Mesopotamia. In: R. Pruzsinszky et al. (ed.), Musiker und Tradierung: Studien zur Rolle von Musikern bei der Verschriftlichung und Tradierung von literarischen Werken (W00 8), Wien, 199-239.

Mielke, D. P. (2006): Inandiktepe und Sarissa: Ein Beitrag zur Datierung althethitischer Fundkomplexe. In: D. P. Mielke et al. (ed.), Strukturierung und Datierung in der hethitischen Archäologie (Byzaz 4), Istanbul, 251-276.

hier, ob das Prinzip bala $\hat{g}$, mit der zugeordneten Gottheit zu kommunizieren und als sein Berater zu fungieren, auf andere Objekte übertragen wurde. 
Mirelman, S. (2014): The Ala-Instrument: Its Identification and Role. In: J. Goodnick Westenholz et al. (ed.), Music in Antiquity: The Near East and the Mediterranean (Yuval 8), Jerusalem, 148-171.

Moran, W. L. (1992): The Amarna Letters, Baltimore - London.

Özgüç, T. (1998): İnandıktepe: Eski Hitit çağında önemli bir kült merkezi, Ankara.

Pruzsinszky, R. (2009): Mesopotamian Chronology of the $2^{\text {nd }}$ Millennium B.C.: An Introduction to the Textual Evidence and Related Chronological Issues (Denkschriften der Gesamtakademie 56/Contributions to the Chronology of the Eastern Mediterranean 22), Wien.

Rashid, S. A. (1984): Musikgeschichte in Bildern. Band 2: Musik des Altertums. Lieferung 2: Mesopotamien, Leipzig.

Richter, Th. (2004): Untersuchungen zu den lokalen Panthea Süd- und Mittelbabyloniens in altbabylonischer Zeit, 2. verb. und erw. Auflage (AOAT 257), Münster.

Römer, W. H. Ph. (2004): Die Klage über die Zerstörung von Ur (AOAT 309), Münster.

Sallaberger, W. (1993): Der kultische Kalender der Ur III-Zeit (UAVA 7/1-2), Berlin u. a.

Schuol, M. (2004): Hethitische Kultmusik: Eine Untersuchung der Instrumental- und Vokalmusik anhand hethitischer Ritualtexte und von archäologischen Zeugnissen (Orient-Archäologie 14), Berlin.

Sefati, Y. (1998): Love Songs in Sumerian Literature: Critical Edition of the Dumuzi-Inanna Songs (Bar-Ilan Studies in Near Eastern Languages and Culture), Ramat-Gan.

Shehata, D. (2009): Musiker und ihr vokales Repertoire: Untersuchungen zu Inhalt und Organisation von Musikerberufen und Liedgattungen in altbabylonischer Zeit (GBAO 3), Göttingen.

Shehata, D. (2014): Sounds from the Divine: Religious Musical Instruments in the Ancient Near East. In: J. Goodnick Westenholz et al. (ed.), Music in Antiquity: The Near East and the Mediterranean (Yuval 8), Jerusalem, 102-128.

Smith, S. (1965): The Art and Architecture of Ancient Egypt, Corrected Reprint, Middlesex u. a.

van Lieven, A. (2008): Native and Foreign Elements in the Musical Life of Ancient Egypt. In: A. A. Both et al. (ed.), Herausforderungen und Ziele der Musikarchäologie (Studien zur Musikarchäologie 6 = Orient-Archäologie 22), Rahden (Westfalen), 155-160.

Veldhuis, N. C. (1997/1998): The Sur ${ }_{9}$-Priest, the Instrument ${ }^{\text {giš }}{ }^{\text {Al-gar-sur }}{ }_{9}$, and the Forms and Uses of a Rare Sign, AfO 44-45, $115-128$.

Volk, K. (1989): Die Balag-Komposition Úru Àm-ma-ir-ra-bi: Rekonstruktion und Bearbeitung der Tafeln 18 (19'ff.), 19, 20 und 21 der späten, kanonischen Version (FAOS 18), Stuttgart.

Volk, K. (2006): Inanas ,Tischlein Deck’ Dich‘, BagM 37, 91-116.

Weeden, M. (2011): Hittite Logograms and Hittite Scholarship (StBoT 54), Wiesbaden.

Young, J. G. (1998): Music in the Aegean Bronze Age, Åström.

Zawadzki, S. (2006): Garments of the Gods: Studies on the Textile Industry and the Pantheon of Sippar according to the Texts from the Ebabbar Archive (OBO 218), Fribourg - Göttingen.

Ziegler, N. (2007): Les musiciens et la musique d'après les archives de Mari (FM 9 = Mémoires de N.A.B.U. 10), Paris. 